\title{
A fuzzy system for detection and classification of textile defects to ensure the quality of fabric production
}

\author{
Iman Subhi Mohammed, Israa Mohammed Alhamdani \\ Department of Computer Science, University of Mosul, Iraq
}

\begin{tabular}{l}
\hline \hline Article Info \\
\hline Article history: \\
Received Des 16, 2018 \\
Revised Apr 16, 2019 \\
Accepted Apr 27, 2019 \\
\hline
\end{tabular}

\section{Keywords:}

Elman neural network Fabric defect detection Fuzzy Logic Gabor filter

\begin{abstract}
The aim of this research focuses on construct a computerized system for textile defects detection. The system merges between image processing methods, statistical methods in addition to the Intelligent techniques via Neural Network and Fuzzy Logic. Gabor filters were used to identify edges and to highlight defective areas in fabric images, then to train the neural network on statistical and geometry features derived from fabric images to form the special neural network distinguish and classify defects into the fourteen categories, which are the most common defects in the textile factory. The proposed work includes two phases. The first phase is to detect the defects in fabrics. The second phase is the classification phase of the defect. At the defect detection stage, a Discrete Cosine Transfer (DCT) converts the images to the frequency domain. Image features then drawn and introduce them to the Elman Neural Network to detect the existence of defects. In the classification stage, the images are converted to the frequency domain by the Gabor filter and then the image features are extracted and inserted into the back propagation network to classify the fabric defects in those images. Fuzzy logic is then applied to neural network outputs and interference values are used in fuzzy logic to increase final discrimination. We evaluate a distinction rate of $91.4286 \%$.After applying the fuzzy logic to neural network output; the discrimination rate was raised to $97.1428 \%$
\end{abstract}

Copyright () 2019 Institute of Advanced Engineering and Science. All rights reserved.

\section{Corresponding Author:}

Israa Mohammed Alhamdani,

Department of Computer Sciences,

Mosul University,

Mosul, Iraq.

Email: esramk65@gmail.com

\section{INTRODUCTION}

Quality control of industrial products has become increasingly important. Quality control is defined as "knowledge of conformity of products to standard specifications and knowledge of the causes of deviation and necessary procedures". The textile industry is an industry that requires quality to meet consumer demand [1]. The main issue here is how fabric screening improves production quality. Under what circumstances? The process of checking the fabrics to this day is a final step before handing the fabrics to the store and then marketing them. This process is still manual by a group of skilled staff with the utmost accuracy from $60 \%$ to $70 \%$ only [2]. To achieve this, the modern textile industry has developed high-speed machines to produce high-quality fabrics in the shortest possible time [3, 4].

The proposed work focuses on solving the problem of textile defects detection manually by automate textile defects via a computerized system. The computerized system is required to check these defects in reliability and flexibility manner. This system merges the image processing methods, statistical methods, intelligent techniques via fuzzy logic and neural network for recognition. As well as to build a live textile database image due to the eliminated existing textile database and the difficulty to get the existing once. The proposed work determined the defective areas in fabric images. The geometrical and statistical 
features were derived to train the neural networks used. The proposed work constructed with two phases. The first phase is to detect the defects while the second classify them. We got $91.4286 \%$ then after applying fuzzy logic to neural network output, the recognition rate was raised to $98.1428 \%$. Rest of this research is structured as the related work in this section the related textile defects system and method were presented, methods of fabric test with various kind. A description of Artificial Intelligent Techniques used in this work also Gabor Filter. Another section deal with the proposed work algorithm, database configuration. Fabric defects stage were the defects is denoted, fabric defects classification, results which are found and discussion, performance efficiency of the proposed work, conclusion, indeed, references. The new idea adapted on this system over other related work is the hyperactive between fuzzy logic with neural network that affects the results in this work.

\section{RELATED WORK}

In order to enhance the quality control of the textile production we construct a computerized system for defects detection and classification. The construction of such a powerful system required deep study on the others methods belong to the textile detection to eliminate their drawback and exceed it in our system. Review of a number of the related work to textile defects detection is disscussed below:

In 2012, researchers Anand H. Kulkarni and Sheetal B. Patil [2] introduced a pattern of detection and identification of defects of tissue based on Gray Level Co-occurrence Matrix (GLCM) as well as a probabilistic neural network. The overall rate of success of tissue selection was $96.6 \%$ and the success rate of defect detection was $91.1 \%$ [5]. Michael K. Nng et al. analyzed paper and tissue patterns of the patterned fabrics in several type. They examined and photographed defects in these fabrics using the convex optimization algorithm their recognition rate ranged between (94.9\% - 99.6\%) [6]. In 2015, Ali Rabhi et al. relied on local homogeneity and neural network to judge defects in tissue images after applying DCT and extracting energy characteristics from images[7], with a detection rate of 97.35\% [8]. Malek use FFT and cross correlation method simultaneously to detect 14 type of defects with accurate results with $100 \%$ recognition rate [9].

M. Handmandulu et.al. Got $70 \%$ rate with 25 textile defect and normal textile images [10]. As presented by P.Y. Kumbhar et al. in a new method was based on the genetic algorithm, the extraction of certain geometrical characteristics also the use of SVM as a classification of tissue defects. The researchers succeeded in classifying $90.0 \%$ of the defects [11]. Soo and Tae based on Wavelet transformation with GMM they get accurate results [12]. In the year 2017, researcher Sunil Bangare et al. suggested a method for detecting tissue defects using RGB, HSV techniques and image processing techniques, and the accuracy of detection was 96.15\% [13]. In the year 2018, Shuang Mei et.al. using Gaussian Pyramid's automatic noise reduction network. The accuracy obtained of the final results reached more than $80.0 \%$ [14].

\section{FABRIC TEST METHODS}

The features extraction methods are classified into three categories they are. The statistical approaches that rely on statistical behavior in areas without defects. Such as statistical moment and cross-correlation [15-17]. The spectral approaches that are used when statistical methods are unable to detect the defects that appear when subtle and very subtle transformations occur. For example, the Gabor filters, FFT and wavelets transform [15-17]. Models based approaches, this model is suitable for fabric images that contain random surface variations as well as random or hand-patterned fabrics. The Gauss Markov Random Field model is one of the methods in this field $[13,16,17]$

\section{ARTIFICIAL INELLIGENT TECHNIQUES}

The speed with which the computer is in its mathematical operations could be used in many non-mathematical tasks as well. Neural networks have been used as an intelligent technique for the detection and classification of defects. The following Neural Network were used in this work.

\subsection{Neural network back propagation (BPNN)}

This is based on training in the error correction pattern. The signal from the output to the input is re-invoked as an learning stage, during which the network weights are calculated, the changes are calculated, and the Mean Square Error(MSE), which results from the difference between the actual output and the desired output, and uses the error to change the weights to gradually reduce the error [18]. 


\subsection{Elman network}

Is one of the recurrent neural networks it is constructed from two-layer with a back propagation feed that was reflected in the first layer re-input to the first layer input. This feature gives the network the possibility to reach the optimal solution with a speed in training and weight control. One of the advantages of the Elman network is that it has a strong dynamic memory, so it is used in different fields such as classification, prediction, dynamic identification systems, and qualitative control [19].

\subsection{Fuzzy neural network}

The neural network can be combined with the logic caused by a number of methods, including the processing of data entering the neural network. The use of the neural network to modify the parameters of the organic functions in the logic, or the neural network outputs before the classification decision is made. Use the threshold value in the rating decision process; this significantly affects the rating ratio for each category and overall system. We may obtain the highest rating of the first defect at a certain threshold limit but not the appropriate limit that gives the highest rating of the defect. Thus, a neural network was proposed in which the threshold role was abolished and replaced by the logic of the reasoned logic called the FPBN. The output of the neural network was used as input to the reasoning logic that was based on specific laws [20].

\section{GABOR FILTER}

The Gabor filters mimic the human brain's ability to recognize the fabric [21] the images were segmented into many scaled images, each containing varying densities on a narrow range of frequency and direction. Gabor filters can be defined as "a set of wavelets, with each wavelet capturing power at a given frequency and direction", so Gabor's two-dimensional efficiency in optimizing the local information of the image, as well as its accessibility [22, 23].

\section{THE PROPOSED WORK ALGORITHM}

The proposed work algorithm in general includes two stages. The first stage is the discovery of defects and their classification into two defective and non-defective categories. The second stage is the classification of these defects into fourteen types. In the first phase, the images were pre-processed by resize the image. Then images were treated with multiple Gabor filters to highlight the existing defects. The resulting image is segmented and the good statistical characteristics for each image are extracted to form the input matrix. This matrix was entered to the Elman Neural Network that was used to classify the textile to non-defective and defective textile. Figure 1 shows the steps of the first stage.

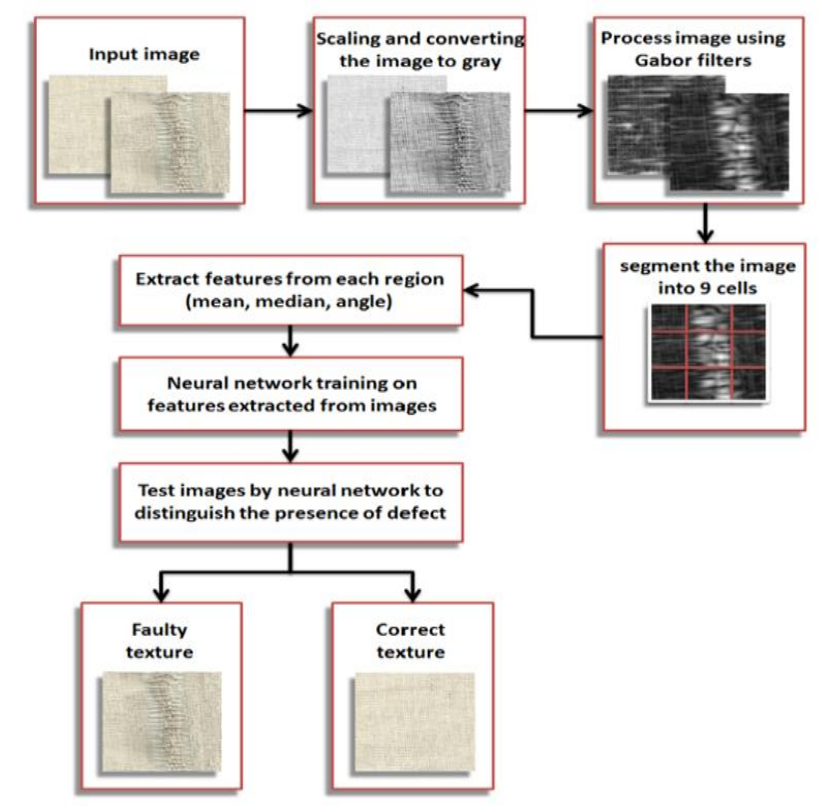

Figure 1. The textile defects detection 
At the second stage, the defective textile images from the first stage are processed then, the defects were determined. Their appropriate statistical features are extracted, and the input matrix is prepared as an input to BPNN. The hybridization between Back Propagation Neural Network and the fuzzy logic is performed to obtain the proposed Fuzzy Back Propagation Neural Network (FBPNN). In FBPNN the output is made fuzzy and represented as input to the fuzzy logic. Fuzzy logic depends on a specific law to make the final classification decision and classify the defects if it is within the used fourteen categories. Figure 2 show these steps.

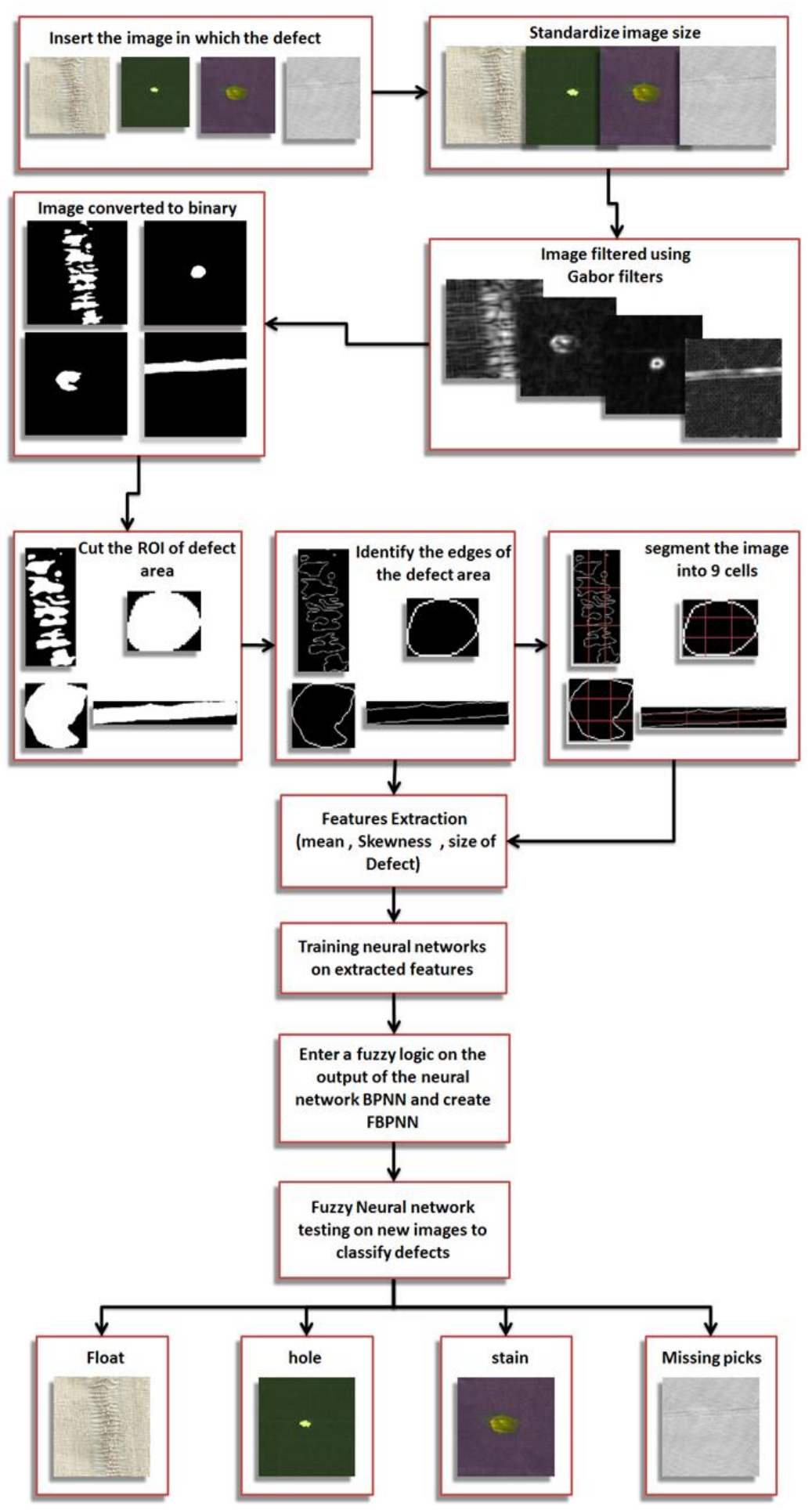

Figure.2. Defects classification stage 
The proposed work details is described as follows:

\subsection{Database configuration}

The fabric images were collected life by a digital camera (samsung NV lens and 8 mega pixel) from a local textile factory. Also, samples of fabrics from the local market. Images were captured by the scanner type (HP) and accuracy (300 dpi). We collect defective and non-defective textile images to be used for our proposed work or any others work. Figure 3 shows samples of database textile images. Five hundred sixty RGB images were collected and saved on a compressed form (PNG). We prefer this from on many image format in order to keep the details of the image despite compression. Twenty hundred eighty image of them were non-defective images and 280 were of the 14 defect types. Twenty image per fourteen defects was collected as a textile database.
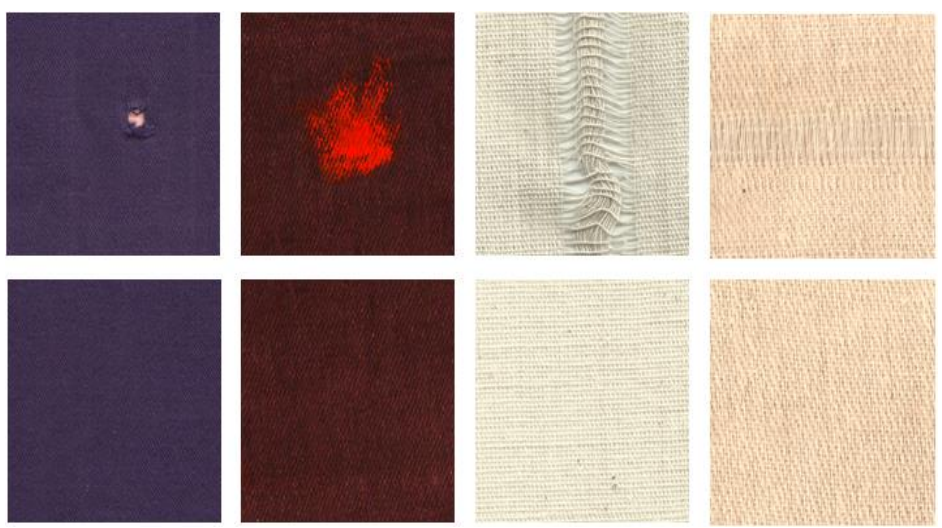

Figure 3. Sample from visual database

\subsection{Textile defects stage}

At this stage, the 560 images are processed. Neural networks were used to distinguish between defective and the non-defective fabric. This process is evaluated as follows:

\subsubsection{Initial processing}

The textile image is entered into the system. It is converted to a gray scale in order to eliminate complex calculations as well as to facilities the deal with the image details. Then this image is treated with Gabor filter the use of this filter reflects better results and describe superior features.

\subsubsection{Image crops and features extraction}

The resulting image from Gabor is segmented into 9 tiles of equal size as shown in Figure 4. For each tile, features were extracted represented by The Ac coefficient (corner), Mean and median were calculated. These values were normalized in a vector representing image characteristics and the formation of this vector is entered into the neural network through the defect detection stage.

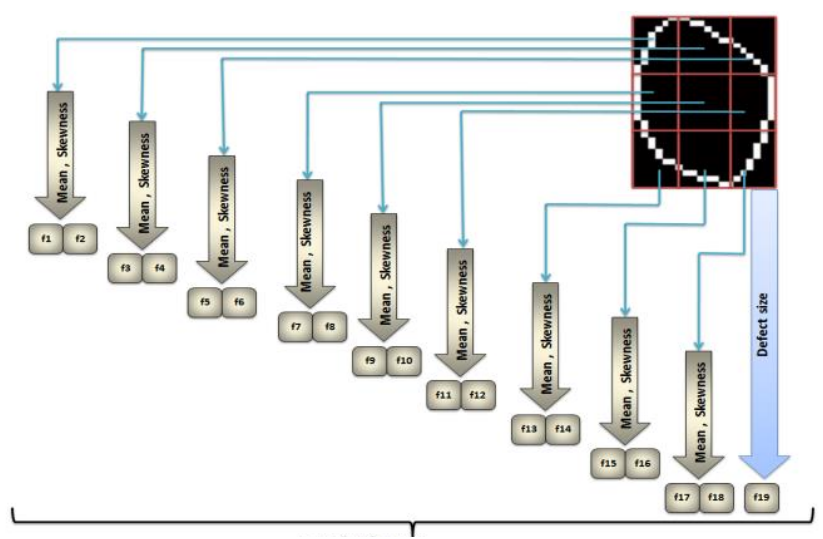

Figure 4. Nine tiles of the defect

A fuzzy system for detection and classification of textile defects to ensure the ... (Iman Subhi Mohammed) 


\subsubsection{Fabric defect detection stage}

The Elman Neural Network is applied to identify and detect where the textile is defect or nondefective. This network consists of an input layer including 21 input neurons, and one hidden layer consisting of about 10 processing neuron. In each of these layers, there is a layer of interconnections that connect each layer to the next layer, in which the weights of each interface are adjusted. The weight is linked to each pair of neurons. The weights represent a weight vector (w1, w2, wn), Where the weight associated with the connection between an input neuron and a processing element or between two treatment elements. The neuron also contains a threshold value (continued activation) that regulates the probability of activation and limits the output of the cell where it makes the output within the field [0, 1] or within the field $[1,1]$. The weights associated with neuronal input determine the probability of activation of the neuron according to (1) [15]:

$$
S U M=\sum_{i=1}^{n} X_{i} W_{i}
$$

Where Xi represents input neuron and Wi-vector weights.

The threshold regulates the response of the neuron to fall within a certain range of predetermined values as shown in (2) [24]:

$$
y=f\left(\sum_{i=1}^{n} X_{i} W_{i}\right)
$$

The (2) shows the y output of the neuron as a function of $f$ activation of the sum of $n+1$ of the balanced input, where $n+1$ corresponds to $n$ of incoming signals. The type of activation function used then calculates the actual output value, and here the threshold function is used as in equation (3) [24]:

$$
f(x)=\left\{\begin{array}{c}
1 \text { if } \sum_{i=1}^{n} X_{i} W_{i}>0 \\
0 \text { if } \sum_{i=1}^{n} X_{i} W_{i} \leq 0
\end{array}\right\}
$$

The defect detection phase also goes through two stages the first is the network training in the characteristics of each image and its expected output. The output used to develop the network efficiency to test and detect the rest images in order to find out whether the tested images is for defective textile or not. The second stage is the test phase via testing the images that not trained to detect if these images are defective or not. The network is then trained on the features this process learn the network to be able to detect if the textile image either defective textile or non-defective. The detection results show that the output is " 0 " if the textile is free of defects or the output is "1" if the textile is defective. Figure 5 describe this process. The training matrix has been configured from 420 to 210 textile images without defect and the other 210 has a defect and a target matrix configuration that corresponds to it.

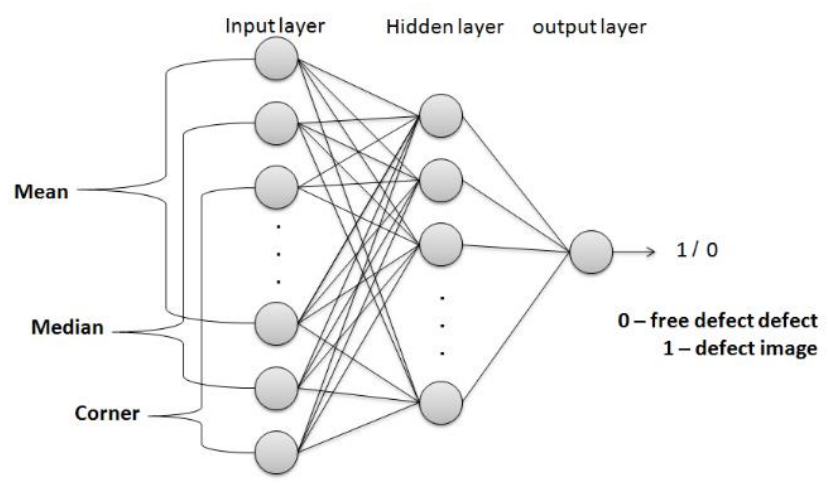

Figure 5. Elman Neural Network for defects detection 


\subsection{Fabric defects classification stage}

At this stage, only 210 defective textile images processed and classified by the Back Propagation Neural Network into fourteen defects as discussed below:

\subsubsection{Isolate the defect area (region of interest ROI)}

After reading and scaling the defective textile image. This image is treated with Gabor filter. Then the resultant gray image is binarized where "0" indicates "black" pixel and " 1 " indicates "white" pixel via fixed threshold [25]. The defect area then eliminated and distinguished this process denotes the automatic cutting algorithm of the defect area. The outcome of these processes is a binary image with a described (ROI).

\subsubsection{Features extraction and defects classification}

The total size of the defect area is calculated and considered as a geometric feature of the image. The perimeter of the defect area is then determined after Canny Operators are applied to the image. The defect image is segmented to nine tiles, then their features were extracted represented by Mean and Skewness is calculated as in (4) [26]. The characteristics of the defect images are arranged in a two-row matrix whose number of rows represent the number of images and the number of columns representing the number of attributes. The total features is $(2 \times 9=18)$ and one for Area so the total features to each defect textile image is equal 19. This matrix is entered into the neural network for classification of defect types.

$$
\text { skewness }=\frac{1}{N}\left(\frac{\sum_{i=1}^{N}\left(X_{i}-\bar{X}\right)^{3}}{\sigma^{3}}\right)
$$

The defects are then classified into 14 categories using the Back Propagation Neural Network that it is trained and then tested.

\subsubsection{Neural network training}

The network is trained on the characteristic vector of the defective images. The purpose of learning the network is that to enforce the network to increase its accessibility to classify defects. Each input vector consists of 19 values representing the mean and the skewness of each cell in addition to the size of the defect area. These features are normalized to the highest value of the nine tiles.

\subsubsection{Neural network for defect classification}

Follow the same steps as the initial process and features extraction to obtain the characteristic vector of the input image. Later, this vector was entered into the neural network to identify the defect type and return to which class it belongs. During the training process, parameter and optimal weights storing on training phase. Then these values were restored for testing phase to test the images which non-trained on the network.

\section{RESULTS AND DISCUSSION}

In order to get the best performance of our system many tries were used with many NN. A number of images were selected for training with minimum error and to reach the optimal weight. The same images also used for testing phase to check the power of the neural network used. The results gained corresponds to each neural network configured the powerful and appropriate network choice. Another sample from the image database that was not used in the training phase were used for the testing phase. To measure the performance of our system two quality measures namely, true value and error value were used they are defined as (5) and (6) respectively [27].

$$
\begin{aligned}
& \text { TR_In order V = A_V (i)/E_V (i) } * 100 \% \\
& \text { ER_V }=\left(100-T R \_V\right) * 100 \%
\end{aligned}
$$

Where A_V represent the number of correctly classified image, E_V represents the entire database image. 


\subsection{Elman neural network}

This network were used for defect detection it was trained on 420 images, including 210 nondefective textile image and anther 210 textile defective image before. A new sample of 140 images from the collected visual database non-trained before were formed to be trained in the Elman Neural Network. These 140 images include 70 of non-defective textile image and 70 defective textile image. This sample was entered into ELMAN network for defect detection. The results of the trained 420 image and non- trained 170 images were described as shown in Table 1. The results for the trained 210 images are equal to $100 \%$ for defective and non-defective image. While the rate for the 70 defective image approximate to $98.57 \%$ and $100 \%$ for the non-defective 70 images. In addition, the total results are about $100 \%$ of the 210 image and $99.28 \%$ of the 140 images. These results at all reflect the superior results and the appropriate selection of the neural network.

Table 1. Results of trained and non-trained images on the Elman NN

\begin{tabular}{lll}
\hline Textile type & 210 trained Image & 70 not trained Image \\
\hline Defect textile & $100 \%$ & $98.5714 \%$ \\
Non defect textile & $100 \%$ & $100 \%$ \\
Total percentage & $100 \%$ & $99.2857 \%$ \\
Time ellipse in second & $0: 00: 46$ & 63.0785 \\
\hline
\end{tabular}

\subsection{BPNN and FBPNN neural network testing stage}

Were applied on feature vector during the test phase to classify the defects type of the 70 images. At the test phase the BPNN train for five images per 14 defects each the accuracy of the defect classification ratio was $91.4286 \%$ with error ratio $8.5714 \%$. In addition, FBPNN train the same images as BPNN its classification ratio was about $97.1428 \%$ with error rate $4.2857 \%$ overall this image set. The results shown in Table 2 configure the power of our proposed neural network denoted by FBPNN over BPNN for both resultant parameters.

Tables 2. Results for test stage by BPNN and FBPNN

\begin{tabular}{lcccc}
\hline Defect Type & \multicolumn{2}{c}{ Accuracy of BPNN } & \multicolumn{2}{c}{ Accuracy of FBPNN } \\
& Accuracy & Error ratio & Accuracy & Error ratio \\
\hline Defect1(Broken ends) & $100 \%$ & $0 \%$ & $100 \%$ & $0 \%$ \\
Defect2 (Broken pattern) & $100 \%$ & $0 \%$ & $100 \%$ & $0 \%$ \\
Defect3 (colored flecks) & $100 \%$ & $0 \%$ & $100 \%$ & $0 \%$ \\
Defect4( Cut \& tear) & $80 \%$ & $40 \%$ & $100 \%$ & $20 \%$ \\
Defect5(Double end) & $100 \%$ & $0 \%$ & $100 \%$ & $0 \%$ \\
Defect6(float) & $100 \%$ & $0 \%$ & $100 \%$ & $0 \%$ \\
Defect7(hole) & $100 \%$ & $0 \%$ & $100 \%$ & $0 \%$ \\
Defect8(Knots) & $80 \%$ & $20 \%$ & $100 \%$ & $0 \%$ \\
Defect9((Missing picks) & $60 \%$ & $40 \%$ & $80 \%$ & $20 \%$ \\
Defect10((ReedM) & $100 \%$ & $0 \%$ & $100 \%$ & $0 \%$ \\
Defect11(slub) & $100 \%$ & $0 \%$ & $100 \%$ & $0 \%$ \\
Defect12(stain) & $80 \%$ & $20 \%$ & $80 \%$ & $20 \%$ \\
Defect13(Untrimm) & $100 \%$ & $0 \%$ & $100 \%$ & $0 \%$ \\
Defect14(Weft Bar) & $100 \%$ & $0 \%$ & $100 \%$ & $0 \%$ \\
Percentage ratio & $91.4286 \%$ & $8.5714 \%$ & $97.1428 \%$ & $4.2857 \%$ \\
\hline
\end{tabular}

\subsection{BPNN and FBPNN neural network training stage}

At the training stage 70 image features vector were trained their results is shown in Table 3 . Fourteen defects classification accuracy was computed at BPNN and FBPNN their results are 99.5238\% and 100\% respectively. This ratio was affected by defect 12 , that it is accuracy ratio approximates to $93.4 \%$ for BPNN but $100 \%$ for FBPNN.

Tables 3. Results for training stage by BPNN and FBPNN

\begin{tabular}{|c|c|c|c|c|c|}
\hline Defect Type & $\begin{array}{l}\text { Accuracy/ } \\
\text { BPNN }\end{array}$ & $\begin{array}{l}\text { Accuracy / } \\
\text { FBPNN }\end{array}$ & Defect Type & $\begin{array}{l}\text { Accuracy/ } \\
\text { BPNN }\end{array}$ & $\begin{array}{l}\text { Accuracy / } \\
\text { FBPNN }\end{array}$ \\
\hline Defect1(Broken ends) & $100 \%$ & $100 \%$ & Defect9((Missing picks) & $100 \%$ & $100 \%$ \\
\hline Defect2 (Broken pattern) & $100 \%$ & $100 \%$ & Defect10((Reed Marks) & $100 \%$ & $100 \%$ \\
\hline Defect3 (colored flecks) & $100 \%$ & $100 \%$ & Defect11(slub) & $100 \%$ & $100 \%$ \\
\hline Defect4( Cut \& tear) & $100 \%$ & $100 \%$ & Defect12(stain) & $93.333 \%$ & $100 \%$ \\
\hline Defect5(Double end) & $100 \%$ & $100 \%$ & Defect13(Untrimmed & $100 \%$ & $100 \%$ \\
\hline Defect6(float) & $100 \%$ & $100 \%$ & Loose threads) & & \\
\hline Defect7(hole) & $100 \%$ & $100 \%$ & Defect14(Weft Bar) & $100 \%$ & $100 \%$ \\
\hline Defect8(Knots) & $100 \%$ & $100 \%$ & Percentage ratio & $99.5238 \%$ & $100 \%$ \\
\hline
\end{tabular}




\section{PERFORMANCE EFFICIENCY OF THE PROPOSED WORK}

In order to measure the enhancement of our proposed work. We compare it with other works in the same field. Despite the difference in visual database size and the techniques used between our system and other related work. Table 4 shows the superiority of our work that reached to $97.1428 \%$ accuracy rate over others work which it is ranged between minimum value $80 \%$ and about $96 \%$ of maximum value.

Table 4. Comparison with related work

\begin{tabular}{|c|c|c|c|}
\hline \multirow{3}{*}{$\frac{\text { Systems }}{\text { Anand H. et al. (2012) [1] }}$} & Method & Visual Database & Accuracy Rate over all DB \\
\hline & GLCM+ Probability & 150 textile image & \\
\hline & Neural Network & $\begin{array}{l}30 \text { textile image free of defects } \\
120 \text { textile image with } 4 \text { type of defects }\end{array}$ & $91.1 \%$ \\
\hline Ali Javed et al. & Regular Band & 78 textile image & \\
\hline (2013) [8] & & $\begin{array}{l}39 \text { textile image free of defects } \\
39 \text { textile image with defects }\end{array}$ & $96 \%$ \\
\hline $\begin{array}{l}\text { P.Y. Kumbhar et al. } \\
\text { (2016) [11] }\end{array}$ & $\begin{array}{l}\text { Genetic algorithm + } \\
\text { SVM }\end{array}$ & ------- & $90 \%$ \\
\hline $\begin{array}{l}\text { Sunil Bangare et. al } \\
\text { (2017) [13] }\end{array}$ & $\begin{array}{l}\text { Image Processing } \\
\text { Technique }\end{array}$ & $\begin{array}{l}52 \text { textile image } \\
26 \text { textile image free of defects } \\
26 \text { textile image with defects }\end{array}$ & $96.15 \%$ \\
\hline $\begin{array}{l}\text { Shuang Mei et. al. (2018) } \\
\text { [14] }\end{array}$ & $\begin{array}{l}\text { Encryption+ Gaussian } \\
\text { Pyramids }\end{array}$ & TELDA database & $80 \%$ \\
\hline $\begin{array}{l}\text { Proposed Approach } \\
\text { (2018) }\end{array}$ & $\begin{array}{l}\text { Gabor filter+ Fuzzy } \\
\text { Back Propagation }+ \\
\text { Elman neural network }\end{array}$ & $\begin{array}{l}560 \text { textile image } \\
280 \text { textile image free of defects } \\
220 \text { image with } 14 \text { type of defects }\end{array}$ & $97.1428 \%$ \\
\hline
\end{tabular}

\section{CONCLUSION}

The proposed work show that the use of spectral filters such as a Gabor filter has greatly helped to obtain good statistical features for the success of the detection process. In addition, Elman and BPNN networks proved their efficiency through their resultant ratios. They show $99.2857 \%$ at the defect detection stage and $91.4286 \%$ in the defects classification stage. As well as the merge between fuzzy logic with a Back Propagation Neural Network that produce the Fuzzy Back Propagation Neural Network FBPNN. The outcome from FBPNN increased the proportion of detection significantly to $97.1428 \%$.

\section{REFERENCES}

[1] Anand H. Kulkarni and Sheetal B.Patil, "Automated Garment Identification and Defect Detection Model Based on Texture Features and PNN," International Journal of Latest Trends in Engineering and Technology (IJLTET), vol. 1, no. 2, 2012.

[2] R. Peng Pai, "Automated Defect Detection for Textile Fabrics Using Gabor Wavelet Networks," Dissertation, The HKU Scholars, The University of Hong Kong, http://hdl.handle.net/10722/51685.[Internet], 2006.

[3] A. P. S. Sawhney, J. B. Price and Calamari, "A Successful Weaving Trial with a Size-Free Cotton Warp," Southern Regional Resrarch Center, Agricultural Research Service, New Orleans, Indian Journal of Fibre \& Textile Research, vol. 29, pp. 117-121, 2004.

[4] Rishav D., Monika A., "Identification Of Defects In Textile Based On Statistical Analysis Of DCT Coefficients Of Textile Images," Aarhat Multidisciplinary International Education Research Journal (AMIERJ), 2012.

[5] Jianyun Ni, Jing Luo, Zaiping Chen and Enzeng Dong, "A Multi Resolution Method for Detecting Defects in Fabric Images Research", Journal of Applied Sciences, Engineering and Technology, Maxwell Scientific Organization, vol. 5, no.5, 2013.

[6] Michael K. Ng, Henry Y. T. Ngan, Xiaoming Yuan, and Wenxing Zhang, "Patterned Fabric Inspection and Visualization by the Method of Image Decomposition," IEEE Transaction on Automation Science and Engineering, vol. 11, no. 3, pp. 943-947, 2014.

[7] Ali Javed, Mirza Ahsan Ullah, and Aziz-ur-Rehman, "Comparative Analysis of Different Fabric Defects Detection Techniques," Image, Graphics and Signal Processing Journal, vol. 1, pp. 40-45, 2013.

[8] Ali Rebhi, Issam Benmhammed, Sabeur Abid, and Farhat Fnaiech, "Fabric Defect Detection Using Local Homogeneity Analysis and Neural Network," Hindawi Publishing Corporation, Journal of Photonics, vol. 9, 2015.

[9] Malek Abdel Salam, "Online Fabric Inspection by Image Processing Technology," Ph.D. Thesis submitted to Haute Alsace University, 2013.

[10] M Hanmandlu, Sujata Dash And D K Choudhur, "Fabric Image Defect Detection by using GLCM and ROSETTA," HIT Transactions, vol. 3, no. 9, 2008

[11] P. Y. Kumbhar, Tejaswini Mathpati, Rohini Kamaraddi and Namrata Kshirsagar, "Textile Fabric Defects Detection and Sorting Using Image Processing," International Journal For Research In Emerging Science And Technology, vol. 3, no. 3, 2016.

[12] Soo Chang Kim and Tae JinKang, "Texture Classification and Segmentation Using Wavelet Packet Frame and Gaussian Mixture Model," Pattern Recognition, vol. 40, no. 4, 2007.

\footnotetext{
A fuzzy system for detection and classification of textile defects to ensure the ... (Iman Subhi Mohammed)
} 
[13] Sunil L. Bangare, N. B. Dhawas, V. S. Taware, S. K. Dighe, P. S. Bagmare, "Implementation of Fabric Fault Detection System Using Image Processing," International Journal of Research in Advent Technology, vol. 5, no. 6, 2017.

[14] Shuang Mei, Yudan Wang, and Guojun Wenm, "Automatic Fabric Defect Detection with a Multi-Scale Convolutional Denoising Autoencoder Network Model," Sensors, 2018.

[15] Ajay Kumar and Hauz Khas, "Computer Vision-based Fabric Defect Detection: A Survey," Department of Electrical Engineering Indian Institute of Technology, 2004.

[16] Mahajan P. M., J. T. Mahajan and Patil P. M, "A Review of Automatic Fabric Defect Detection Techniques," Advances in Computational Research, vol.1, no. 2, pp. 18-29, 2004.

[17] Priyanka M. Shanbhag, Manish P. Deshmukh and Shekhar R. Suralka, "Overview: Methods Of Automatic Fabric Defect Detection," Global Journal Of Engineering, Design \& Technology, vol. 1, no. 2, pp. 42-46, 2012.

[18] Tom Mitchell, Machine Learning, published by McGraw Hill, 1997.

[19] Zhiqiang Zhang, Shangce Gao, Gang Yang, Fangjia Li and Zheng Tang, "An Algorithem of Supervised Learning for Elman Neural Network," International Journal of Innovative Computing, Information and Control ICIC, International, vol. 5, no. 10(A), pp. 2997-3011, 2009.

[20] MathWorks Inc., "Fuzzy Logic Toolbox TM ", User's Guide R2014a, 2014.

[21] S. Arivazhagan, L. Ganesan, and S. Bama, "Fault Segmentation in Fabric Images Using Gabor Wavelet Transform," Journal of Machine Vision and Applications, vol. 16, no. 6, pp. 356-363, 2006.

[22] Dengsheng Zhang, Aylwin Wong, Maria Indrawan, Guojun Lu, "Content-Based Image Retrieval Using Gabor Texture Features," Proceeding of First IEEE Pacific-Rim Conference on Multimeadia, pp. 392-395, 2000.

[23] Omar Sultan Al-Kadi, "Agabor Filter Texture Analysis A for Histopathological Brain Tumour Sub Type Discrimination," ISESCO JST journal, vol.12, no. 22, 2017.

[24] Ajay Kumar, "Neural Network Based Detection of Local Textile Defects," The Journal Of Pattern Recognition, The Pattern Recognition Society, vol. 36, pp. 1645-1659, 2003.

[25] Maya R. Gupta , Nathaniel P. Jacobson and Eric K. Garcia, "OCR Binarization and Image Pre-Processing for Searching Historical Documents," The Journal Of The Pattern Recognition, vol. 40, pp.389-397, 2007.

[26] David P. Doane and Lori E. Seward, "Measuring Skewness: A Forgotten Statistic?," Journal of Statistics Education, Oakland University, vol. 19, no. 2, 2011.

[27] S. G. HOGGAR, Mathematics of Digital Images Creation, Compression, Restoration, Recognition, USA: Cambridge University Press, New York, 2006 